\title{
ORTHOGONAL SPHERES, LIGHT CONES AND CAUSALITY IN MINKOWSKI SPACE
}

\author{
ROBERT J. LOW
}

Communicated by Gregory L. Naber

Abstract. We describe a curious relationship between orthogonal spheres and causal relationships in Minkowski space.

MSC: 83A05

Keywords: Causality, light cone, Minkowski space, orthogonal spheres

\section{Introduction}

We are going to consider causal relationships in Minkowski space $\mathbb{M}^{4}$, equipped with the usual Minkowskian coordinates $(t, x, y, z)$ and inner product $\langle$,$\rangle with sig-$ nature $(+,-,-,-)$. In particular, we are going to consider the relationship between how two points are causally related with how their light cones intersect in a surface of constant time, and see how orthogonality of the resulting spheres is involved.

First, we recall that two spheres are orthogonal if the vectors from the centre of each to a (and hence every) point of intersection are orthogonal. This is clearly equivalent to the sum of the squares of the radii of the spheres being the square of the distance between their centres.

Now, let us consider the growth of two spheres each of whose radii grows in time, where one starts as a point and the other initially has radius $R$.

Then, for $p \in \mathbb{E}^{3}$, where $\mathbb{E}^{3}$ is the usual three dimensional Euclidean space, we denote by $S_{p}(r)$ the sphere of radius $r$ with centre $p$. Let $p, q \in \mathbb{E}^{3}$, let the distance between $p$ and $q$ be $d$, and let $R>0$. Then we have the fundamental geometric result

Lemma 1. The spheres $S_{p}(|t|)$ and $S_{q}(|t+R|)$ intersect orthogonally for some $t$ iff $d \geq R / \sqrt{2}$.

Proof: The spheres intersect orthogonally when $t$ satisfies

$$
d^{2}=t^{2}+(t+R)^{2}
$$


i.e.,

$$
t=\frac{R \pm \sqrt{2 d^{2}-R^{2}}}{2}
$$

which is possible if and only if $d \geq R / \sqrt{2}$.

\section{Causal Relationships in Galilean and Minkowski Space-Time.}

In Galilean space-time $\mathbb{G}^{4}$ with coordinates $(t, x, y, z)$, causal relationships are particularly simple. The two events $P$ and $Q$ are timelike separated if there is a surface of constant $t$ which separates $P$ and $Q$; if there is, then $Q$ is to the future of $P$ if the $t$-coordinate of $Q$ is greater than that of $P$, and to the past of $P$ if the $t$-coordinate of $Q$ is less than that of $P$. Otherwise, $P$ and $Q$ have the same $t$-coordinate, and are simultaneous.

The situation is somewhat more subtle in $\mathbb{M}^{4}$. Now, if $P$ and $Q$ are any distinct points, then in some Minkowskian coordinate system there is a surface of constant $t$ which separates $P$ and $Q$. The notion of causal separation can be considered in various ways (see [1] for an extremely detailed discussion of the geometry of Minkowski space-time), of which two are described below.

Denote by $V$ the vector connecting $P$ to $Q$. Then the points $P$ and $Q$ are spacelike separated if $\langle V, V\rangle\langle 0$, null separated if $\langle V, V\rangle=0$, and timelike separated if $\langle V, V\rangle>0$. In the latter two cases, $Q$ is to the future of $P$ if in some (and hence any) Minkowskian coordinate system the $t$-coordinate of $Q$ is greater than that of $P$.

There is a more immediate visual characterization obtained by using the idea of the light cone. The light cone of $P$ consists of the set of all points connected to $P$ by a null vector, and is denoted $N(P)$; the future light cone, $N^{+}(P)$, is the set of those points whose $t$ coordinate is greater than that of $P$ and the past light cone, $N^{-}(P)$, all those whose $t$ coordinate is less. We can then see that $Q$ is to the future of $P$ if it lies inside $N^{+}(P)$, the past of $P$ if it is inside $N^{-}(P)$ and is spacelike separated from $P$ if it is outside $N(P)$.

Then we ask: if we look at the intersections of the light cones of a pair of points with a surface of simultaneity, can we use orthogonality of spheres to characterize the causal relationship between the two points? 


\section{Causal Relations From Light Cone Intersections}

We now consider two points $P, Q \in \mathbb{M}^{4}$, and denote the vector connecting $P$ to $Q$ by $V$.

The first and most obvious relationship is that if $V$ is null, ie if $\langle V, V\rangle=0$, so that $P$ and $Q$ lie on each other's light cone, then in every surface of constant $t$, the light cones of $P$ and $Q$ intersect tangentially.

Next, let us consider the case where $V$ is spacelike, so $\langle V, V\rangle<0$. Furthermore, let $T$ be an arbitrary timelike vector. Abusing notation slightly by identifying points and their position vectors, we denote by $\Sigma_{t}$ the hyperplance orthogonal to $T$ and passing through $P+t T$.

Then $N(P)$ and $N(Q)$ intersect $\Sigma_{0}$ in a point and a sphere, $P$ and $\Sigma_{0} \cap N(Q)$ respectively, where $P$ is outside $\Sigma_{0} \cap N(Q)$. Without loss of generality, we can assume that $Q$ is to the past of $\Sigma_{0}$. Denoting by $R$ the radius of $\Sigma_{0} \cap N(Q)$ and by $d$ the distance between $P$ and the centre of $\Sigma_{0} \cap N(Q)$, we then see that as we consider the intersection of $N(P)$ and $N(Q)$ with $\Sigma_{t}$ we are in precisely the situation of Lemma 1 with $d>R$, and so $d>R / \sqrt{2}$. Thus the light cones of $P$ and $Q$ will intersect orthogonally in $\Sigma_{t}$, where

$$
t=t_{ \pm}=\frac{R \pm \sqrt{2 d^{2}-R^{2}}}{2} .
$$

Furthermore, one can see that these surfaces of simultaneity do not separate $P$ and $Q$. This follows either from a direct argument about the values of $t$, taking into account whether the $Q$ is to the future or past of $\Sigma_{0}$, or by noting that if $N(P)$ intersected $N(Q)$ in some point $X$ on a spacelike surface separating $P$ and $Q$ then there would be a causal chain from $P$ to $X$ and from $X$ to $Q$, contradicting the assumption that $P$ and $Q$ are not causally related [2].

Indeed, by considering the values of $t$, we observe that one of the $\Sigma_{t}$ lies to the future of both $P$ and $Q$, while the other lies to the past of both.

The final case to consider is when $\langle V, V\rangle>0$ ie $V$ is timelike. We let $\Sigma_{t}$ be the hyperplane orthogonal to $V$ and passing through $P+t V$. Then we see that the null cones $N(P)$ and $N(Q)$ intersect $\Sigma_{t}$ in concentric circles, and so either have empty intersection or coincide: they never intersect orthogonally.

We can thus characterize causal relationhips in $\mathbb{M}^{4}$ as follows.

Theorem 2. Let $P, Q \in \mathbb{M}^{4}$. Then

1. $P$ and $Q$ are null separated if the null cones of $P$ and $Q$ intersect tangentially in every surface of simultaneity. 
2. $P$ and $Q$ are spacelike separated iffor every timelike vector $T$ there is a pair of surfaces of simultaneity each orthogonal to $T$ in which the light cones of $P$ and $Q$ intersect orthogonally. Furthermore, these surfaces divide $\mathbb{M}^{4}$ into three regions. Two of them contain neither of $P$ and $Q$, and third lies between the other two and contains both of $P$ and $Q$.

3. $P$ and $Q$ are timelike separated if they are not null separated and there exists a timelike vector $T$ such that the light cones of $P$ and $Q$ never intersect orthogonally in any surface of simultaneity orthogonal to $T$.

This is not quite satisfactory, however. It does not address the possibility that for a pair of timelike separated points, $P$ and $Q$ there may by a timelike vector $T$ and a surface of simultaneity in which $N(P)$ and $N(Q)$ intersect orthogonally. In fact, this can always be done.

Theorem 3. If $P, Q \in \mathbb{M}^{4}$ then there exists a timelike vector $T$ such that there is a surface of simultaneity orthogonal to $T$ in which $N(P)$ and $N(Q)$ intersect orthogonally.

Proof: Denote by $V$ the vector connecting $P$ to $Q$, and let $\langle V, V\rangle=R^{2}$. If $T$ is any unit timelike vector, i.e., $\langle T, T\rangle=1$, then $Q=R^{\prime} T+d S$ where $\langle S, T\rangle=0$ and $\langle S, S\rangle=-1$ so that $S$ is a unit spacelike vector orthogonal to $T$. Then in the $\Sigma_{0}$, the surface of simultaneity through $P$ and orthogonal to $T$, we see that $R^{\prime}$ is the radius of $N(Q) \cap \Sigma_{0}$ and $d$ is the distance between $P$ and the centre of $N(Q) \cap \Sigma_{0}$. Furthermore

$$
R^{2}=R^{\prime 2}-d^{2}
$$

For appropriate choices of $T, R^{\prime}$ and $d$ can take on any values subject only to the preceding equation; so in particular it is possible to choose $T$ such that $R^{\prime} / \sqrt{2} \leq$ $d<R^{\prime}$.

But where does this surface lie? Since for any surface of simultaneity to the past or future of both $P$ and $Q$, one light cone is entirely within the other, the surface must separate $P$ and $Q$. Furthermore, when $d=R^{\prime} / \sqrt{2}$ there is just one surface, then as $d$ increases towards $R$ a pair of parallel surfaces splits apart and approach the points $P$ and $Q$ in the limit. We note that dually, for spacelike separated $P$ and $Q$ we can choose the timelike vector $T$ to make $d$ any value greater than $R$, and that the pair of surfaces orthogonal to $T$ in which $N(P)$ and $N(Q)$ intersect orthogonally approach $P$ and $Q$ as $d$ approaches $R$ (where now $\langle V, V\rangle=-R^{2}$, and separate off to future and past infinity as $d$ increases.

This allows us to formulate the following rather more symmetrical and pleasing statement 
Theorem 4. Let $P, Q \in \mathbb{M}^{4}$, and let $\Sigma$ be a surface of simultaneity such that $N(P)$ and $N(Q)$ intersect orthogonally in $\Sigma$. Then $P$ and $Q$ are timelike separated if $\Sigma$ separates $P$ and $Q$, and spacelike separated if $P$ and $Q$ are on the same side of $\Sigma$.

\section{Comments}

There remains something slightly mysterious about the case when $P$ and $Q$ are timelike separated. For some choices of the normal, there is surface of simultaneity separating $P$ and $Q$ in which the null cones $N(P)$ and $N(Q)$ intersect orthogonally, but not for all. One might consider complexifying, but in this case we although gain a choice of surface with orthogonally intersecting light cones, it now no longer separates points at all, so cannot be used to classify the causal relationship.

Note, finally, that although the above discussion was for the physical case of three spatial dimensions, the arguments work in example the same way for any number of spatial dimensions greater than or equal to two.

\section{Bibliography}

\section{References}

[1] Naber G., The Geometry of Minkowski Spacetime, $2^{\text {nd }}$ Edn, Springer, London 2012.

[2] Penrose R., Techniques of Differential Topology in Relativity, SIAM, Philadelphia 1972.

Robert J Low

School of Computing, Electronics

and Mathematics

Coventry University

Priory Street

Coventry CV1 5FB

UK

E-mail address:mtx 014 @ coventry . ac.uk 